\title{
Relevance of DPP IV/CD26 among the Gut-brain Axis during Experimental Colitis ${ }^{\dagger}$
}

\author{
Lara Batičić Pučar, ${ }^{\mathrm{a}}$ Dijana Detel, ${ }^{\mathrm{a}}$ Sunčica Buljević, ${ }^{\mathrm{a}}$ Ester Pernjak Pugel, ${ }^{\mathrm{b}}$ \\ Natalia Kučić, ${ }^{c}$ and Jadranka Varljen ${ }^{\mathrm{a}, *}$ \\ ${ }^{\mathrm{a}}$ Department of Chemistry and Biochemistry, School of Medicine, University of Rijeka, \\ Braće Branchetta 20, HR-51000 Rijeka, Croatia \\ ${ }^{\mathrm{b}}$ Department of Histology and Embriology, School of Medicine, University of Rijeka, \\ Braće Branchetta 20, HR-51000 Rijeka, Croatia \\ ${ }^{\mathrm{c}}$ Department of Physiology and Immunology, School of Medicine, University of Rijeka, \\ Braće Branchetta 20, HR-51000 Rijeka, Croatia
}

RECEIVED DECEMBER 10, 2010; REVISED JULY 7, 2011; ACCEPTED JULY 26, 2011

\begin{abstract}
Inflammatory bowel diseases (IBD) represent a group of chronic conditions of the gastrointestinal tract of unknown etiology. Latest knowledge accentuates the bidirectional connection between the central and enteric nervous systems. An important role of peptidases has been proposed in maintaining the homeostasis in the gut. One of them is dipeptidil-peptidase IV (DPP IV/CD26), a multifunctional glycoprotein found in both soluble and membrane-bound form in living organisms. In order to evaluate the relevance of DPP IV/CD26 among the gut-brain axis, a TNBS (Crohn-like) model of colitis has been induced in CD26 deficient and wild type mice. Results of this study showed that CD26 deficient mice show specificity in histological damage compared to wild type mice. A decreased DPP IV/CD26 activity was found in serum, colon and brain in wild type mice with colitis, while CD26 protein expression was increased in colon of those mice. DPP IV/CD26-like activity was decreased only in colon of CD26 deficient mice. Changes occurring during inflammatory processes in colon reflected on investigated parameters in brain. Therefore, our results indicate the importance of the gut-brain axis in the pathogenesis of IBD. (doi: 10.5562/cca1813)
\end{abstract}

Keywords: dipeptidyl-peptidase IV, CD26 molecule, CD26 deficient mice, inflammatory bowel diseases, TNBS-colitis, gut-brain axis

\section{INTRODUCTION}

Inflammatory bowel diseases (IBD) represent a group of idiopathic, chronic inflammatory conditions that could affect different segments of the gastrointestinal tract, with numerous complications and extraintestinal manifestations. ${ }^{1}$ Two main pathomorphological entities of IBD are Crohn's disease (CD) and ulcerative colitis (UC), sharing some similarities, but also having individual characteristics in disease manifestations. The etiology of IBD, despite of many investigations, still remains unknown. ${ }^{2}$ It is generally accepted that IBD occurs in genetically susceptible individuals, under influence of environmental and microbiological factors, as an overexpressed immunological response to antigens of unknown origin. ${ }^{3}$ Latest knowledge regarding IBD pathogenesis includes the influence of neuroimmunological parameters, which are driven by the hypothesis that neurogenic inflammation could be involved in inflammatory processes in the gut. ${ }^{4}$ Given the connection of enteric and central nervous system and the bidirectional communication between the gut and the brain, a term "gut-brain-axis" has been introduced and proposed as an important aspect in the etiology of IBD. ${ }^{5}$

In order to elucidate the pathogenesis of IBD, different animal models of gastrointestinal inflammation have been developed. ${ }^{6}$ One of the most accepted experimental model of Crohn-like-colitis is the trinitrobenzenesulfonic acid-induced (TNBS) colitis, which resembles many histological, immunological and clinical manifestations of $\mathrm{CD}$ in humans. ${ }^{7}$ Animal models of IBD have given insight in different processes at the molecular level which lead to development of inflammatory changes and have revealed the importance of many molecules involved in IBD etiology. ${ }^{8}$

\footnotetext{
$\dagger$ Presented at the $10^{\text {th }}$ Congress of the Croatian Society of Biochemistry and Molecular Biology held in Opatija, Croatia, September 15-18, 2010.

* Author to whom correspondence should be addressed. (E-mail: vjadran@medri.hr)
} 
In the last decade, proteases have been proposed as important factors in inflammatory processes, given their ability to metabolize different biologically active substrates involved in maintaining the integrity of the mucosal barrier. ${ }^{9}$ One of them is dipeptidyl-peptidase IV, known also as CD26 molecule (DPP IV/CD26). DPP IV/CD26 is a highly glycosylated type II transmembrane sialoglycoprotein comprising two identical subunits of approximately $110 \mathrm{kDa} .{ }^{10} \mathrm{DPP}$ IV/CD26 was originally characterized as a T-cell differentiation antigen and was reported to be expressed on various cell types, having multiple functions in various biological processes, including immunological regulations. ${ }^{11}$ A soluble form circulating in body fluids is found in living organisms. As a serine protease, it possesses a specific peptidase function with unique features in substrate processing: it cleaves dipeptides from the $\mathrm{N}$-terminus of polypeptides where proline or alanine is at the penultimate position. Since N-termini containing Xaa-Pro are not easily cleaved by other proteases, the action of DPP IV/CD26 is a rate-limiting step in the degradation of many polypeptides. ${ }^{12}$

Different cytokines, chemokines and neuropeptides involved in inflammatory events are found among DPP IV/CD26 substrates. ${ }^{13}$ Our hypothesis was that CD26 deficient mice would express differences in inflammatory processes and disease manifestations. This study was undertaken in order to have an insight in histological and biochemical alterations at local and systemic levels during development and resolution of colitis under conditions of DPP IV/CD26 deficiency in comparison with wild type mice. Furthermore, we wanted to evaluate changes in DPP IV/CD26 and DPP IV/CD26-like activity at local and systemic levels, likewise DPP IV/CD26 protein expression in targeted tissues during colitis development and resolution. Given the potential importance of the gut-brain axis in the development of inflammatory processes, experiments were focused on changes occurring at local site of inflammation - in colon, and in the central nervous system - in brain.

\section{EXPERIMENTAL}

\section{Experimental Animals}

This study was performed using two mice strains: wild type mice strain $\mathrm{C} 57 \mathrm{BL} / 6$ and mice with inactivated gene for molecule CD26 (C57BL/6 Jbom-ob, CD26 ${ }^{-1}$ ), generated on a $\mathrm{C} 57 \mathrm{BL} / 6$ genetic background. CD26 deficient mice were kindly provided by Dr. Didier Marguet, Centre d'Immunologie Marseille-Luminy, France. Generation of CD26 deficient mice has been described previously. ${ }^{14}$ Male, $8-10$ week old mice were used in this study. Animals were housed and bred under standard conditions at the Central Animal Facility of the School of Medicine, University of Rijeka. Laboratory animals were housed in plastic cages, fed with standard pellet food (MK, Complete Diet for Laboratory Rats and Mice, Slovenia) and given tap water ad libitum. They were maintained under a 12/12 hours dark/light cycle at constant temperature $(20 \pm 1){ }^{\circ} \mathrm{C}$ and humidity (50 \pm 5$) \%$. Each study group comprised 8-10 animals. Animal handling, experimental procedures and anesthesia were performed in accordance with the general principles contained in the Guide for the Care and Use of Laboratory Animals (National Academic Press). The Ethical Committee of the School of Medicine, University of Rijeka, approved all experimental procedures.

\section{Colitis Induction}

Crohn-like-colitis (TNBS-colitis) was induced by rectal administration of $5 \%$ (mass concentration) 2,4,6-trinitrobenzenesulfonic acid solution (TNBS, SigmaAldrich, Germany) dissolved in $50 \%$ ethanol (Kemika, Croatia). Each animal received $0.1 \mathrm{~mL}$ of TNBSethanol solution, using a vinyl catheter that was positioned $4 \mathrm{~cm}$ from the anus, as described previously. ${ }^{7}$ Two control groups of mice were used for each mice strain. Control mice underwent identical procedures, but were instilled equal volumes of saline $(\mathrm{NaCl}, \gamma=0.9 \%)$ or ethanol solution. Administration of TNBS, saline or ethanol solution was performed in mice anesthetized with ketamine/xylazine.

\section{Experimental Procedures}

Animals were sacrificed by cervical dislocation after 2 , 7, 15 and 30 days upon administration of TNBS, saline or ethanol solution. Peripheral blood samples were taken and serum samples were collected by centrifugation at $3000 \mathrm{rpm}$ for 10 minutes. Colons were freed from adhering tissue and macroscopic changes were noted. The colon lumen was carefully washed with ice-cold saline and its weights and lengths was measured. One part of the colon was used for histological analyses while the remaining segments of the colon were slit open longitudinally and the mucosa was scraped using a glass microscope slide. Colonic mucosal samples were prepared from obtained mucosal scrapings according to Ahnen et al. ${ }^{15}$ Obtained mucosal aliquot samples were stored at $-80{ }^{\circ} \mathrm{C}$ until further analyses.

Brains were separated immediately after sacrifice, washed in ice-cold saline and frozen in liquid nitrogen then stored at $-80^{\circ} \mathrm{C}$ until further analyses. Brains were homogenized on ice in a volume of $0.4 \mathrm{~mL}$ of $0.1 \mathrm{M}$ TRIS-HCl buffer $(\mathrm{pH}=8)$. Homogenates were then centrifuged at $14000 \mathrm{rpm}$ for 20 minutes at $+4{ }^{\circ} \mathrm{C}$. Resulting supernatants were measured for total protein concentrations according to the method of Bradford et al. ${ }^{16}$ 


\section{Histological Analyses}

Colon tissues were collected and fixed in $4 \%$ formalin for $24 \mathrm{~h}$. Samples were processed and embedded in paraffin wax. Two-micrometer sections were stained with hematoxylin and eosin for histological analyses. An experienced pathologist blinded to treatment allocation scored microscopical changes, including overall severity of damage, number of crypts of Lieberkühn and their depth and width. Microscopic analysis was carried out using an Olympus BX40 microscope (Tokyo, Japan) and the Pulnix TMC 76S digital camera (Tokyo, Japan). Analyses of digital images were performed using Issa software package (VAMS, Zagreb, Croatia).

\section{DPP IV/CD26 Activity Assay}

The DPP IV/CD26 (in C57BL/6) and DPP IV/CD26like enzymatic activities (in CD26 deficient mice) in serum, brain and colon homogenates were measured according to the protocol of Kreiser et al..$^{17}$ by detection of free 4-nitroaniline in an assay mixture containing $0.1 \mathrm{M}$ Tris- $\mathrm{HCl}$ buffer $(\mathrm{pH}=8)$, and $2 \cdot 10^{-3} \mathrm{M}$ Gly-Pro $p$-nitroanilide as the substrate (Sigma-Aldrich, Germany) in a total volume of $0.20 \mathrm{~mL}$. The reaction was stopped by addition of $0.80 \mathrm{~mL}$ of sodium acetate buffer $\left(1 \mathrm{~mol} \mathrm{dm}^{-3}, \mathrm{pH}=4.5\right)$ after 30 minutes of incubation at $37^{\circ} \mathrm{C}$. The amounts of hydrolyzed substrate were measured in duplicates spectrophotometrically at $\lambda=450 \mathrm{~nm}$ using a Varian Cary UV/Vis Spectrophotometer. Enzyme activities are expressed according to the Nomenclature Committee of the International Union of Biochemistry. Serum DPP IV/CD26 and DPP IV/CD26like enzyme activities are expressed as amount of substrate hydrolyzed per liter of serum $\left(n k a t \mathrm{~L}^{-1}\right)$. DPP IV/CD26 and DPP IV/CD26-like enzyme activities in brain and colon homogenates are expressed as the amount of substrate hydrolyzed per mg of protein (nkat $\mathrm{mg}^{-1}$ of protein).

\section{CD26 Western Blot Analysis}

Brain and colon samples for Western blot analyses of CD26 protein expression were homogenized on ice using RIPA lysis buffer including inhibitors of proteases and phosphatases (Santa Cruz Biotechnology Inc., CA). Homogenates were then centrifuged at $14000 \mathrm{rpm}$ for 20 minutes at $+4{ }^{\circ} \mathrm{C}$. Resulting supernatants were measured for total protein concentrations according to the method of Bradford et al. ${ }^{16}$ All samples were heated for 5 min at $95^{\circ} \mathrm{C}$ in a sample buffer. Prestained molecular weight markers (Kaleidoscope Prestained Standards, BIO-RAD and Fermentas Life Sciences PageRuler ${ }^{\mathrm{TM}}$ ) were used as standards. Equal amounts of total proteins (50 $\mu \mathrm{g}$ per lane) were separated by sodium dodecyl sulphate-polyacrylamide gel electrophoresis on $10 \%$ gels. Samples were electrophorezed at $50 \mathrm{~V}$ for $6 \mathrm{~h}$ on ice.
Proteins were transferred from the polyacrylamide gels to polyvinylidenedifluoride membranes by electroblotting in a semi-dry transfer apparatus at 0.22 $\mathrm{mA}$ for $45 \mathrm{~min}$. Membranes were blocked for 2 hours at $4{ }^{\circ} \mathrm{C}$ in phosphate-buffered saline containing $0.05 \%$ Tween 20 (PBS-T, Amersham Biosciences, USA), containing $5 \%$ (mass concentration) nonfat milk powder (Santa Cruz Biotechnology Inc., CA). Membranes were treated overnight with primary anti-CD26 antibody (Santa Cruz Biotechnology Inc., CA, 1:200 in blocking buffer). Blots were washed three times for $15 \mathrm{~min}$ in PBS-T. As a secondary antibody, horseradish peroxidase-conjugated mouse-anti-rabbit IgG in a dilution of $1: 2000$ (Santa Cruz Biotechnology Inc., CA) was used. After the second washing procedure, CD26 molecule $(110 \mathrm{kDa})$ was detected by chemiluminescent Amersham ECL-plus Western blotting detection reagents (Amersham, Little Chalfont, UK), which enabled visualization of bends after exposure to photosensitive films (AGFA Ortho CP-G plus). Equal total protein loading was ensured with use of the primary mouse $B$-actin antibody (Chemicon International, USA), in a dilution of $1: 40000$, and secondary horseradish peroxidaseconjugated goat-anti-mouse $\operatorname{IgG}$ in a dilution of $1: 2000$ (Santa Cruz Biotechnology Inc., CA).

\section{Statistical Analyses}

Results are expressed as mean \pm standard deviation. Statistical comparisons were made using STATISTICA version 8.0 (StatSoft Inc., TULSA, USA). Testing differences between groups has been performed using ANOVA and post-hoc Scheffe test. The level of $P<0.05$ was defined as statistically significant.

\section{RESULTS AND DISCUSSION}

TNBS-induced colitis in mice is an experimental model of IBD which shares many similarities with $\mathrm{CD}$ in humans at molecular levels and in clinical manifestations of disease and therefore represents a valuable tool in IBD research. ${ }^{7}$ Macroscopic signs of inflammation characteristic for TNBS-induced colitis comprise marked oedema and mucosal thickening, which are accompanied by increased colon weight. ${ }^{18}$ Results of our study are in agreement with these observations. The presence of an accentuated oedema in the colon was determined, with marked inflammatory changes that were most conspicuous the second day following TNBS-ethanol administration. Therefore, the second day after colitis induction was classified as acute phase of colitis, which is in agreement with previously reported findings. ${ }^{7}$ Shortening and thickening of the colon was also observed in mice which received TNBS-ethanol solution. Intrarectal application of TNBS-ethanol solution in mice 
induces a transmural, granulomatous inflammation with infiltration of inflammatory cells mainly located in the distal part of the colon. ${ }^{7}$ Histopathological changes observed in colon tissue sections of both wild type and CD26 deficient mice confirmed the presence of inflammatory processes and accomplishment of colitis induction. It should be emphasized that to date, no TNBSinduced colitis in CD26 deficient mice was described in available literature to our knowledge, and scarce results are published regarding investigation using this mice strain.

Like macroscopic changes, microscopic changes were also most prominent in the acute phase of colitis. Patohystological analyses confirmed the presence of inflammatory changes in the colon of mice that received TNBS-ethanol solution. Partly necrotic mucosa, largely penetrated with granulocytes and disintegrated leukocytes is visible in Figure 1. Inflammatory changes did not overtake the entire mucosa, in most analyzed colon samples a part of the colonic mucosa was preserved with physiological appearance of crypts. Analyses of larger number of colonic sections revealed differences in inflammatory manifestations among two analyzed mice strains. In CD26 deficient mice, ulcerations were mostly localized in a certain part of the mucosal surface, but in a number of mice, a transmural inflammation was observed (Figure 2A). On the other hand, no transmural inflammations were observed in wild type animals, but inflammatory changes affected the entire colonic circumference with very little or no areas of preserved mucosa (Figure 2B).

Results of histomorphometrical analyses also confirmed the presence of inflammatory changes in both mice strains which received TNBS-ethanol solution. Number of crypts of Lieberkühn per mm of mucosa, and their depth and width for different groups of both mice strains at given days of experiment were measured (data not shown). Statistical analyses of obtained results among both control groups of animals did not reveal statistically significant changes in observed parameters, nor at different days of sacrifice. In both mice strains with induced colitis, a statistically significant $(P<0.05)$ decrease in number of crypts of Lieberkühn per mm of mucosa was observed in the acute phase of colitis. Changes persisted even during tissue healing in CD26 deficient mice. The width of crypts of Lieberkühn was increased in the acute phase of colitis in both mice strains, but it took longer to achieve physiological values in wild type mice. Furthermore, the depth of crypts of Lieberkühn was decreased in acute colitis in both mice strains. All those changes represent consequences of inflammatory processes in the colon which include mucosa thickening and formation of oedema due to TNBS-ethanol-induced tissue damage.

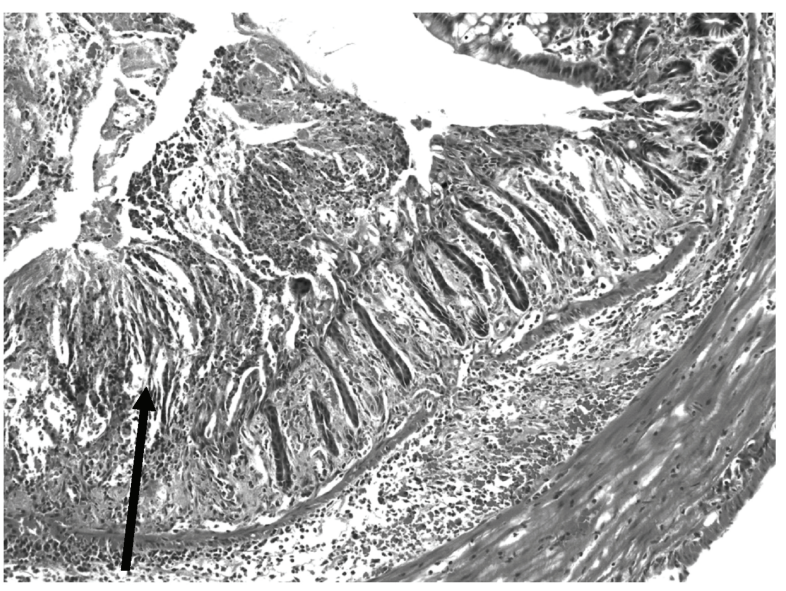

Figure 1. Inflammatory changes in distal colon of C57BL/6 mice in the acute phase of colitis, two days after administration of TNBS-ethanol solution. Colon sections $(2 \mu \mathrm{m})$ were stained with hematoxylin and eosin and examined for pathologic changes. The arrow indicates partly necrotic mucosa, largely penetrated with granulocytes and disintegrated leukocytes. The original magnification was $20 \mathrm{x}$.

Analyses of DPP IV/CD26 activity and protein expression were performed at systemic and local levels, in the serum and among the gut-brain axis respectively, in wild type mice. Since we wanted to evaluate whether in conditions of DPP IV/CD26 deficiency other DPP IV/CD26-like enzymes could partially take over its enzymatic function, DPP IV/CD26-like systemic and local activities were determined in CD26 deficient mice. Results of serum DPP IV/CD26 activity in wild type mice with induced colitis compared to control groups are presented on Figure 3A. A statistically significant decrease $(P<0.05)$ in serum DPP IV/CD26 activity was observed starting in the acute phase of colitis and achieving physiological values after disease healing. Our results accord with previously published results that included determination of serum DPP IV/CD26 activity in patients with IBD and other chronic diseases. ${ }^{19-22}$
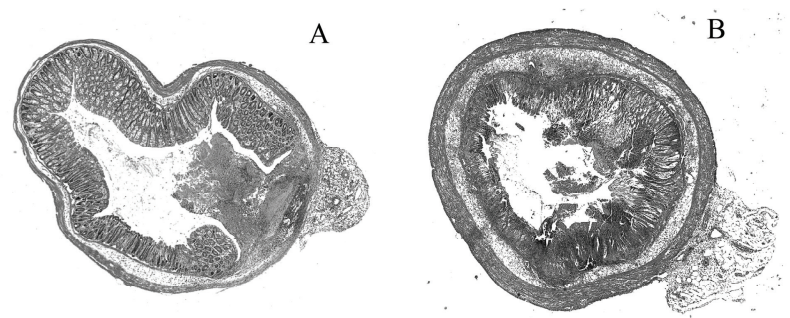

Figure 2. Strain-characteristic inflammatory changes in distal colon of $\mathrm{CD}_{2} 6^{-1-}$ (A) and $\mathrm{C} 57 \mathrm{BL} / 6$ mice (B) in the acute phase of colitis, two days after administration of TNBSethanol solution. Colon sections $(2 \mu \mathrm{m})$ were stained with hematoxylin and eosin and examined for pathologic changes. The original magnification was $4 \mathrm{x}$. 


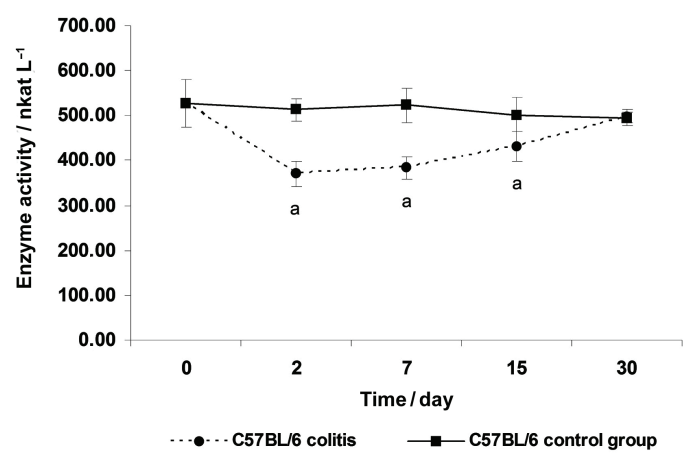

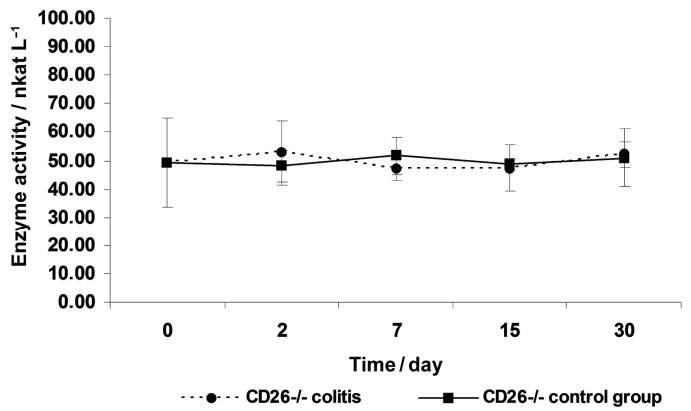

Figure 3. Serum DPP IV/CD26 activity in C57BL/6 mice (A) and serum DPP IV/CD26-like activity in $\mathrm{CD} 26^{-1-}$ mice (B) during colitis development and resolution compared to control group. Statistically significantly different values (a) compared to control group $(P<0.05)$. 0 - control group, physiological condition; 2, 7, 15, 30 days after administration of TNBSethanol solution (colitis group) or ethanol solution (control group).

Therefore, a clinical relevance of serum DPP IV/CD26 activity as a marker of disease severity in IBD was proposed. ${ }^{21}$ Its role in inflammatory events includes degradation of biologically active substrates which have a crucial role in inflammation. Our results are in accordance with the observation that serum DPP IV/CD26 activity correlates inversely with disease severity in patients with IBD. ${ }^{21}$

In order to evaluate changes in serum DPP IV/CD26-like activities during colitis development and healing, CD26 deficient mice were analyzed. Results of our study show that CD26 deficient mice express approximately $10 \%$ of total serum DPP IV/CD26 activity detected in wild type mice. Figure $3 \mathrm{~B}$ shows results of serum DPP IV/CD26-like activity in CD26 deficient mice with induced colitis compared to their control groups. It could be seen that no statistically significantly differences were found in serum DPP IV/CD26-like activity between groups of CD26 deficient animals with colitis and control animals. Therefore, the significance of DPP IV/CD26 over DPP IV/CD26-like serum activity is proposed.
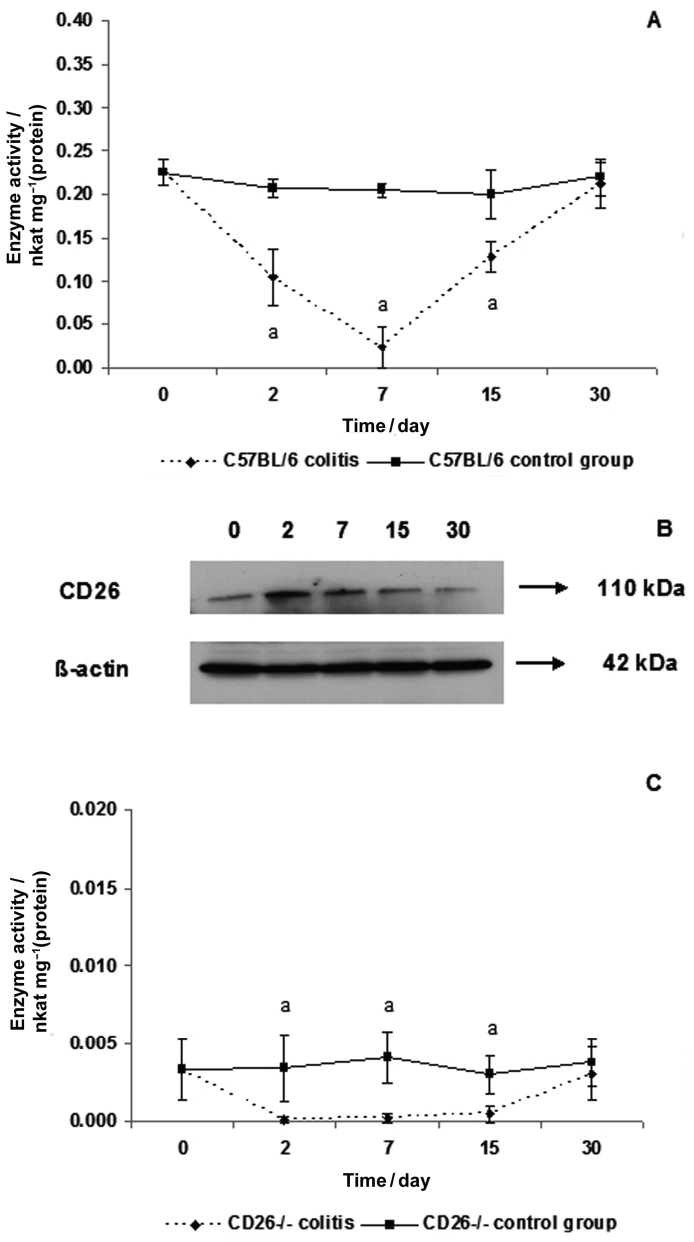

Figure 4. DPP IV/CD26 activity in colon of $\mathrm{C} 57 \mathrm{BL} / 6$ mice (A) and DPP IV/CD26-like activity in colon of $\mathrm{CD} 26^{-1-}$ mice (C). CD26 protein expression in colon of C57BL/6 mice during colitis development and resolution compared to control group (B). Statistically significantly different values (a) compared to control group $(P<0.05) .0$ - control group, physiological condition; 2, 7, 15, 30 days after administration of TNBS-ethanol solution (colitis group) or ethanol solution (control group).

In the last decade, growing scientific evidence emphasizes neuroimmunomodulation as an important factor in the occurrence of inflammation. ${ }^{23}$ Due to the causal connection between central and enteric nervous system, the term gut-brain axis has been proposed. ${ }^{5}$ DPP IV/CD26 has previously been proven to play an important role in metabolism of important bioactive neuroand immunopeptides, as well as in the costimulation of immune cells. ${ }^{11}$ Given its localization in the nervous system and among the digestive tract, likewise on the surface of important inflammatory cells, ${ }^{24}$ our aim was to investigate changes in DPP IV/CD26 activity and protein expression at sight of inflammation and reveal if those changes reflect on examined parameters in the brain. 
Our results showed an accentuated decrease in DPP IV/CD26 activity in the inflamed colon in wild type animals compared to their control groups (Figure 4A). On the other hand, Western blotting technique revealed an increased CD26 protein expression in the acute phase of disease, as shown on Figure 4B. This phenomenon could, besides a regulatory mechanism at the enzymatic level, also be partially explained as a compensatory mechanism, since a part of the decreased DPP IV/CD26 activity in the colon of wild type mice is a consequence of severe mucosal damage induced by TNBS-ethanol application. Therefore, enhanced CD26 protein expression in the acute phase of disease could represent an attempt to accomplish a compensatory role due to damaged DPP IV/CD26 conformation and improper enzyme activity in inflamed tissue. An enhanced DPP IV/CD26 mRNA production has previously been shown under inflammatory environment. ${ }^{25}$ Consequently, a higher CD26 protein expression could be expected in inflammation. Our results are in agreement with previously reported observations regarding enhanced CD26 protein production in inflamed tissue.

Investigations on DPP IV/CD26-like activity in the colon of CD26 deficient mice shown that, in physiological conditions, CD26 deficient mice express less than $2 \%$ of total DPP IV/CD26 activity detected in the colon of wild type mice. Statistically significantly $(P<0.05)$ decreased DPP IV/CD26 activity in inflamed colon homogenates was also found in CD26 deficient mice compared to their controls (Figure 4C). However, this could also partially be a consequence of tissue damage and not only an intrinsic regulation mechanism which downregulates the activity of DPP IV/CD26-like enzymes in inflammatory processes.

Interesting results were found when analyzing DPP IV/CD26 activity in brain homogenates during colitis development and resolution in wild type mice. It was found that DPP IV/CD26 activity in brain is statistically significantly decreased $(P<0.05)$ in the acute phase of colitis compared to control groups (Figure 5A), while the activity of DPP IV/CD26-like enzymes does not change (Figure 5C). On the other hand, CD26 protein expression, as confirmed by Western blot technique (Figure 5B) remains constant. A possible explanation of this phenomenon could be a regulatory mechanism which control DPP IV/CD26 activity in brain, independently of its protein expression. Likewise, since there is no local inflammation in the brain which could destroy DPP IV/CD26 enzymatic function like in the inflamed colon, there is no need for a potential compensatory mechanism which would try to compensate lower DPP IV/CD26 activity by its higher protein expression. Therefore, lower DPP IV/CD26 activity and its unchanged protein expression in the brain most probably represents an intrinsic regulatory mechanism at the enzymatic level.
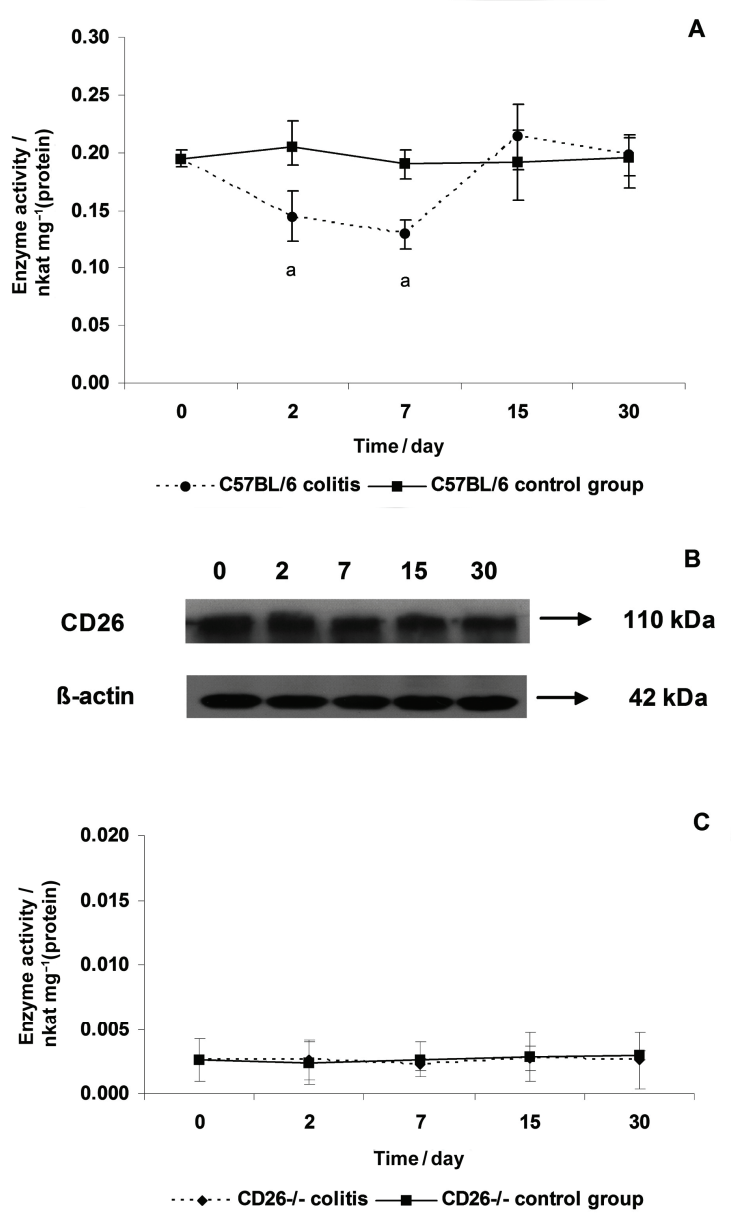

Figure 5. DPP IV/CD26 activity in brain of C57BL/6 mice (A) and DPP IV/CD26-like activity in brain of CD26 $6^{-/-}$mice (C). CD26 protein expression in brain of C57BL/6 mice during colitis development and resolution compared to control group (B). Statistically significantly different values (a) compared to control group $(P<0.05) .0$ - control group, physiological condition; 2, 7, 15, 30 days after administration of TNBS-ethanol solution (colitis group) or ethanol solution (control group).

Moreover, results of this study indicate that changes which occur during inflammatory processes at local level, in the colon, exhibit a reflection on investigated parameters in the central nervous system. Therefore, a decreased DPP IV/CD26 activity in the brain is most probably causally connected with its changes in the colon during inflammatory events. Hence, our results indicate the importance of the gut-brain axis in the pathogenesis of IBD. This study reveals new data about DPP IV/CD26 activity and protein expression in a model of Crohn-like colitis in mice. Likewise, due to very little available results of colitis investigation under conditions of CD26 deficiency, our study gives new insights in inflammatory manifestations induced by TNBS-ethanol administration in CD26 deficient mice. 
Inhibition of DPP IV/CD26 has been proposed as potential therapy for chronic inflammatory diseases. ${ }^{26}$ This hypothesis is supported by higher values of antiinflammatory cytokines obtained in CD26 deficient mice. ${ }^{27}$ Furthermore, it has recently been shown that inhibition of DPP IV/CD26 partially ameliorates colitis in mice. ${ }^{28}$ IBD is characterized by imbalances in innate and acquired immune response, where two dysregulated $\mathrm{T}$ cell subsets are crucial: activated effector $\mathrm{T}$ cells and regulatory $\mathrm{T}$ cells. They are characterized by a strong expression of DPP IV/CD26, which has been confirmed to play a significant role in the control of immune activation and in regulating cellular communication by hydrolyzing bioactive polypeptides. ${ }^{29}$ Positive results in the regulation of immune and non-immune processes in IBD have been proved in vitro as well in vivo with DPP IV/CD26 and alanyl-aminopeptidase inhibitors. ${ }^{30}$

Nevertheless, results of our study showed that CD26 deficient mice are not protected from chemically induced Crohn-like colitis, but exhibit some differences in histological damage compared to wild type mice. Otherwise, CD26 deficient mice show a normal phenotype and behavior as wild type mice, but are known to posses and enhanced insulin secretion and improved glucose tolerance. ${ }^{14}$ This knowledge lead to development of DPP IV/CD26 inhibitors in the therapy of diabetes mellitus type II which are already in clinical use. ${ }^{31-33}$ However, further studies should be done in order to test if DPP IV/CD26 inhibition could be an acceptable therapeutic approach in chronic inflammatory disease alleviation, as new drug candidates for the treatment of diseases associated with an imbalanced $\mathrm{T}$ cell response.

\section{CONCLUSIONS}

This study reveals new data about DPP IV/CD26 activity and protein expression in a model of TNBS (Crohnlike) colitis in mice. Due to meager available results of colitis investigation under conditions of CD26 deficiency, we established a TNBS-ethanol induced colitis in CD26 deficient mice. Our study gives new insights in Crohn-like colitis inflammatory manifestations in CD26 deficient mice. Results of our study showed that CD26 deficient mice are not protected from chemically induced Crohn-like colitis, but show specificity in histological damage compared to wild type mice. A decreased DPP IV/CD26 activity was found in serum, colon and brain in $\mathrm{C} 57 \mathrm{BL} / 6$ mice with colitis, while DPP IV/CD26-like activity is decreased only in colon of CD26 deficient mice. An enhanced protein expression of CD26 molecule was found in colon of wild type mice in the acute phase of colitis. It was noticed that changes occurring during inflammatory processes in the colon, reflect on investigated parameters in the central nervous system. Therefore, our results indicate the importance of the gut-brain axis in the pathogenesis of IBD.

Acknowledgements. This study was supported by the Croatian Ministry of Science, Education and Sports (grant No. 062-0061245-0213). We gratefully acknowledge Dr. Didier Marguet (Centre d'Immunologie Marseille-Luminy, France), for providing CD26 deficient mice. Many thanks to professor Siniša Volarević, PhD, head of the department of Molecular Medicine and Biotechnology and professor Stipan Jonjić, $\mathrm{PhD}$, head of the department of Histology and Embriology, School of Medicine, University of Rijeka, for allowing us to complete a part of experiments using the equipment at their departments.

\section{REFERENCES}

1. S. B. Hanauer and D. W. Hommes, Expert Rev. Clin. Immunol. 6 (2010) 499-500.

2. D. Q. Shih and S. R. Targan, Curr Gastroenterol Rep. 11 (2009) 473-480.

3. R. S. Blumberg, Dig. Dis. 27 (2009) 455-464.

4. Y. Takami, C. R. Mantyh, T. N. Pappas, T. Takahashi, K. Koda, and M. Miyazaki, Hepatogastroenterology 56 (2009) 682-686.

5. J. A. Romijn, E. P. Corssmit, L. M. Havekes, and H. Pijl, Curr Opin. Clin. Nutr. Metab. Care 11 (2008) 518-521.

6. H. H. Uhlig and F. Powrie, Eur. J. Immunol. 39 (2009) 20212026.

7. F. Scheiffele and I. J. Fuss, Current Protocols in Immunology 2002, Chapter 15, Unit 15.19.

8. S. Wirtz and M. F. Neurath, Adv. Drug Deliv. Rev. 59 (2007) 1073-1083.

9. A. Ravi, P. Garg, and S. V. Sitaraman, Inflamm. Bowel. Dis. 13 (2007) 97-107.

10. M. D. Gorrell, X. M. Wang, J. Park, K. Ajami, D. M. Yu, H. Knott, D. Seth, and G. W. McCaughan Adv. Exp. Med. Biol. 575 (2006) 45-54.

11. M. Vanderheyden, J. Bartunek, M. Goethals, S. Verstreken, A. M. Lambeir, I. De Meester, and S. Scharpe, Clin Chem Lab Med 47 (2009) 248-252.

12. M. D. Gorrell, Clin. Sci. 108 (2005) 277-292.

13. R. Mentlein, Regul. Pept. 85 (1999) 9-24.

14. D. Marguet, L. Baggio, T. Kobayashi, A. M. Bernard, M. Pierres, P. F. Nielsen, U. Ribel, T. Watanabe, D. J. Drucker, and N. Wagtmann, Proc. Natl. Acad. Sci. U. S. A. 97 (2000) 68746879 .

15. D. J. Ahnen, N. A. Santiago, J. P. Cezard, and G. M. Gray, J. Biol. Chem. 257 (1982) 12129-12135.

16. M. M. Bradford, Anal. Biochem. 72 (1976) 248-254.

17. W. Kreisel, R. Heussner, B. Volk, R. Buchsel, W. Reutter, and W. Gerok, FEBS Lett. 147 (1982) 85-88.

18. L. Camoglio, A. A. te Velde, A. de Boer, F. J. ten Kate, M. Kopf, and S. J. van Deventer, Eur. J. Immunol. 30 (2000) 1486-1495.

19. M. Hildebrandt, M. Rose, J. Ruter, A. Salama, H. Monnikes, and B. F. Klapp, Scand. J. Gastroenterol. 36 (2001) 1067-1072.

20. H. Kobayashi, O. Hosono, T. Mimori, H. Kawasaki, N. H. Dang, H. Tanaka, and C. J Morimoto, Rheumatol. 29 (2002) 18581866.

21. J. Varljen, B. Mijandrušić Sinčić, L. Batičić, N. Varljen, D. Detel, and A. Lekić, Croat. Chem. Acta 78 (2005) 427-432.

22. N. Busso, N. Wagtmann, C. Herling, V. Chobaz-Peclat, A. Bischof-Delaloye, A. So, and E. Grouzmann, Am. J. Pathol. 166 (2005) 433-442. 
23. L. Ohman and M. Simren, Nat. Rev. Gastroenterol. Hepatol. 7 (2010) 163-173

24. E. Matteucci and O. Giampietro, Curr. Med. Chem. 16 (2009) 2943-2951.

25. E. Nemoto, S. Sugawara, H. Takada, S. Shoji, and H. Horiuch, Infect. Immun. 67 (1999) 6225-6233.

26. U. Aytac and N. H. Dang, Curr. Drug Targets: Immune, Endocr. Metab. Disord. 4 (2004) 11-18.

27. M. S. Geier, D. Tenikoff, R. Yazbeck, G. W. McCaughan, C. A. Abbott, and G. S. Howarth, J. Cell Physiol. 204 (2005) 687-692.

28. R. Yazbeck, G. S. Howarth, M. S. Geier, H. U. Demuth, and C. A. Abbott, Front. Biosci. 13 (2008) 6850-6858.
29. S. Ansorge, U. Bank, A. Heimburg, M. Helmuth, G. Koch, J. Tadje, U. Lendeckel, C. Wolke, K. Neubert, J. Faust, P. Fuchs, D. Reinhold, A. Thielitz, and M. Tager, Clin. Chem. Lab. Med. 47 (2009) 253-261.

30. U. Bank, U. R. Bohr, D. Reinhold, U. Lendeckel, S. Ansorge, P. Malfertheiner, and M. Tager, Front. Biosci. 13 (2008) 36993713.

31. I. De Meester, S. Scharpe, and A. M. Lambeir, Clin. Chem. Lab. Med. 47 (2009) 245-247.

32. D. M. Riche, H. E. East, and K. D. Riche, Am. J. Med. Sci. 337 (2009) 321-328.

33. N. I. Siddiqui, Mymensingh Med. J. 18 (2009) 113-124. 